\title{
Neurofilament Light Chain: A Specific Serum Biomarker of Axonal Damage Severity in Rat Models of Chemotherapy-Induced Peripheral Neurotoxicity
}

Cristina Meregalli 1 , Giulia Fumagalli 1, Paola Alberti 1, Annalisa Canta 1, Alessia Chiorazzi 1 ,

Laura Monza 1, Eleonora Pozzi 1, Valentina Alda Carozzi 1, Kaj Blennow 23 , Henrik Zetterberg 2

345 , Guido Cavaletti 6, Paola Marmiroli 1

1. Experimental Neurology Unit, School of Medicine and Surgery; NeuroMI (Milan Center for Neuroscience), University of Milano-Bicocca, Monza, MB, Italy.

2. Department of Psychiatry and Neurochemistry, The Sahlgrenska Academy at the University of Gothenburg, Mölndal, Sweden.

3. Clinical Neurochemistry Laboratory, Sahlgrenska University Hospital, Mölndal, Sweden.

4. Department of Neurodegenerative Disease, UCL Institute of Neurology, Queen Square, London, UK.

5. UK Dementia Research Institute at UCL, London, UK.

6. Experimental Neurology Unit, School of Medicine and Surgery; NeuroMI (Milan Center for Neuroscience), University of Milano-Bicocca, Monza, MB, Italy. guido.cavaletti@unimib.it.

\section{Corresponding author:}

Dr. Cristina Meregalli, School of Medicine and Surgery, Experimental Neurology Unit and NeuroMI (Milan Center for Neuroscience), University of Milano-Bicocca, v. Cadore 48, 20900 Monza (MB), Italy; e-mail address: cristina.meregalli@unimib.it Tel: +390264488232

Keywords: neurofilament light chain, Simoa, chemotherapy-induced peripheral neurotoxicity (CIPN), axonal degeneration 


\begin{abstract}
Chemotherapy-Induced Peripheral Neurotoxicity (CIPN) is a severe and long-lasting side effect of anticancer therapy, which can severely impair patients' quality of life. It is a sensory and lengthdependent neuropathy, which predominantly affects large myelinated fibers. Easy and reliable monitoring of CIPN in patients is still an unmet clinical need. Since increasing clinical evidence supports the potential use of neurofilament light chain (NfL) as a biomarker of axonal injury, in this study we measured serum NfL levels in animals chronically treated with cisplatin (CDDP) and paclitaxel (PTX), two antineoplastic drugs with different neuronal targets. Wistar rats were treated with CDDP $(2 \mathrm{mg} / \mathrm{kg}$ i.p. twice/week for 4 weeks) or PTX (10 mg/kg i.v. once/week for 4 weeks). Repeated serum NfL quantification was obtained using the Single Molecule Array (Simoa) technology. The onset and progression of peripheral neurotoxicity were evaluated through neurophysiology, morphological assessments and intraepidermal nerve fibers density quantification. Our results showed that serum NfL measurements correlated with the severity of axonal damage. In fact, both treatments induced serum NfL increase, but higher levels were evidenced in PTX-treated animals, compared with CDDP-treated rats, affected by a milder neurotoxicity. Notably, also the timing of the NfL level increase was associated with the severity of morphological and functional alterations of axonal structure. Therefore, NfL could be a useful biomarker for axonal damage in order to follow the onset and severity of axonal degeneration and possibly limit the occurrence of serious PNS disease.
\end{abstract}

\title{
INTRODUCTION
}

Chemotherapy-Induced Peripheral Neurotoxicity (CIPN) is the most prevalent neurological side effect in patients undergoing cancer treatment and may lead to a decline in their daily activities and quality of life (Velasco et al., 2009; Cavaletti et al., 2015a; Cavaletti et al., 2015b). Unfortunately, two of the most effective agents against solid tumors, cisplatin (CDDP) and paclitaxel (PTX), are neurotoxic for a large cohort of long survival patients by inducing a primary neuronopathy or a length-dependent axonopathy, respectively (Carozzi et al., 2015).

Nowadays, it is difficult to assess objectively in a simple way the course of CIPN in the clinical setting. This is a relevant issue since CIPN would be crucial in order to prevent an irreversible and permanent damage.

An emerging concept regards the possible use of neurofilaments (Nfs) as biomarkers of axonal injury due to their exclusive expression in axons where they have a critical role in structural stability. They are 
released into extracellular fluid upon axonal injury and they can be detected in cerebrospinal fluid and blood (Yuan et al., 2012). Nf subunits are biologically classified according to their molecular weight as Nf light (NfL, $68 \mathrm{kDa}$ ), Nf medium (NfM, $160 \mathrm{kDa}$ ) and Nf heavy (NfH, $205 \mathrm{kDa}$ ) (Perrot et al., 2008). Over the last decades, an increased concentration of NfL due to neurodegeneration has been documented in several neurological diseases of the central nervous system (CNS) (Perrot et al., 2009; Novakova et al., 2017; Khalil et al., 2018).. In a previous studies, we demonstrated that NfL was a useful biomarker of axonal degeneration in a rat model of vincristine-induced peripheral neurotoxicity (Meregalli et al., 2018). In the present study, we have extended our evaluations to CDDP- and PTX-treated animals in order to correlate repeated serum NfL dosage to the different mechanisms and severity of neurotoxicity.

\section{MATERIALS AND METHODS}

The studies were approved by the Animal Care and Use Committee of the University of Milano-Bicocca and adhered to all guidelines set forth in the Guide for the Care and Use of Laboratory Animals (Office of Laboratory Animal Welfare) as well as to the Italian D.L.vo n. 26/2014 in compliance with the European Union directive 2010/63/UE.

Two experiments were performed: in the first one, adult female Wistar rats ( $\mathrm{n}=8 /$ group, Envigo, Udine, Italy) were randomized in control (CTRL) and CDDP-treated groups $(2 \mathrm{mg} / \mathrm{kg}$, Accord Healthcare Limited, Middlesex, UK; intraperitoneal injections twice a week for 4 weeks). In the second experiment, CTRL rats and PTX-treated animals $(10 \mathrm{mg} / \mathrm{kg}$ LC laboratories, Woburn, MA, USA; intravenous injections via the tail vein once a week for 4 weeks) were compared.

Neurophysiological examinations (the sensitive action potential amplitude) on caudal nerves were performed after 2 weeks of treatment and at the end of the treatment, as previously described (Meregalli et al., 2018). At the end of the treatment period, morphological analyses on caudal nerves and intraepidermal nerve fiber (IENF) density on skin samples were examined (Chiorazzi et al., 2018; Canta et al., 2016).

Serum Nfl concentration was measured in the Clinical Neurochemistry Laboratory at the University of Gothenburg, Mölndal, Sweden, using an in-house Simoa NfL assay on an HD-1 Analyzer (Quanterix, Billerica, MA), as previously described in detail (Rohrer et al., 2016). All experiments were performed on one occasion, using one batch of reagents. Intra-assay coefficients of variation for quality control samples with $\mathrm{NfL}$ concentrations of $22.6 \mathrm{pg} / \mathrm{mL}$ and $50.2 \mathrm{pg} / \mathrm{mL}$ were below $10 \%$. 
A two sided Mann-Whitney $U$ test was used, and continuous variables were described by the median and $25 \%$ and $75 \%$ percentile (significance level set at $\mathrm{p}<0.05$ ). Statistical analyses were conducted using GraphPad Prism4 statistical package (GraphPad Software, San Diego, CA).

\section{RESULTS AND DISCUSSION}

The lack of a specific biomarker for a direct measure of peripheral nerve damage is a limiting factor in early detection of the axonal degeneration in patients treated with antineoplastic drugs. Up to now, neurofilament (NfL) proteins are promising emerging blood biomarker candidates for axonal degeneration in several neurological disorders mainly affecting the CNS, such as Huntington's disease (Byrne et al., 2017), multiple sclerosis, Alzheimer disease and frontotemporal dementia (Disanto et al., 2017; Mattsson et al., 2017; Rohrer et al., 2016). Conversely, a few studies analyzed serum NfL in patients with peripheral nervous system (PNS) diseases, such as inherited peripheral neuropathies (Sandelius et al., 2018), acquired peripheral neuropathies (Mariotto et al., 2018) and vasculitic neuropathy (Bischof et al., 2018), indicating an increase in concentration compared to the healthy subjects. Given our previous results showing convincing data of serum NfL measure as an axonal damage marker in vincristine CIPN animal model (Meregalli et al., 2018), we here extended these preliminary data in PTX- and CDDP-treated rats, demonstrating that serum NfL levels correlates to neurophysiology and neuropathology findings of neurotoxicity.

According to our data, several features of CIPN development were confirmed at the end of the experiments in both studies performed with PTX and CDDP by different analyses. In particular, a reduction of the small unmyelinated fibers was detected through the IENF density analysis in both groups of treatment compared to control rats (CTRL median=30.83 [IQR 28.3-31.07] fibers/mm, CDDP 21.97 [20.27-23.18] fibers/mm p<0.001; CTRL 30.29 [28.55-36.44] fibers/mm, PTX 19.49 [19.05-22.06] fibers/mm $\mathrm{p}<0.001$ ), confirming the impairment of fibers primarily linked to painful sensation.

Of particular importance, the neurophysiological examination on caudal nerves showed a significant reduction in sensory potential amplitude in PTX-treated animals compared with control rats from the second week of treatment $(p<0.001)$ until the end of the experiment $(p<0.0001)$; whereas a mild difference was detected in CDDP-treated animals compared to their control arm only at the end of the treatment $(\mathrm{p}<0.05)($ Fig1).

Serum NfL concentration was measured using the most sensitive method available to date (Kuhle et al., 2016). The results showed a significant increase in both groups of treatment compared with control 
animals, but the concentration in PTX-treated animals was higher than in CDDP-treated ones from the first week of treatment (Fig2), indicating a correlation between these concentrations and the severity of the axonal damage. These data are in line with our previous results (Meregalli et al., 2018) and they are expected since the neurofilament proteins, the major constituent of the neuronal cytoskeleton, are exclusively released after neuroaxonal injury (Kuhle et al., 2015). It is likely that the concentrations are elevated in the animals with severe neuropathy induced by PTX due to the early strong axonal damage, whereas the NfL levels measured in CDDP-treated rats are lower than PTX-treated animals, showing a progressive increase of the concentration, which corresponds to a minor impairment of peripheral nerves during the ongoing neurotoxicity.

The increase of NfL levels in blood samples was previously noted in various preclinical studies of neurological diseases. For example, a recent work of Soylu-Kucharz and co-workers, showed NfL concentration in cerebrospinal fluid (CSF) and blood as a convincing accessible murine marker for monitoring the progression in Huntington's disease, in correlation with the pathology (Soylu-Kucharz R et al., 2017). Furthermore, Bacioglu and collaborators described an increase of NfL levels in CSF and blood samples in mouse models of tauopathy, synucleinopathies and $\beta$-amyloidosis (Bacioglu et al., 2016). Likewise, Brureau et al. showed serum NfL increase in CSF which correlated with ongoing neuronal damage in cerebral cortex and hippocampus in an inducible transgenic mouse model of neurodegeneration (Brureau et al., 2017).

Remarkably, these findings seem to reflect the prominent axonal loss on caudal nerves collected at the end of the treatment and the presence of several degenerated fibers in PTX-treated animals (Fig3), which correlates with high levels of serum NfL concentrations. These data suggest that the mechanism underlying PTX-induced neurotoxicity involves a direct action of this compound against peripheral nerves, resulting in severe fiber degeneration (Roytta M and Raine, 1986). In contrast, regarding CDDPtreated animals, only a few degenerated fibers were observed in caudal nerves; whereas the analysis on DRG showed a contest of sensory neuronopathy/ganglionopathy with a reduction in soma, nucleus and nucleolus size (CTRL 656.4 [448.5-1344] $\mu^{2}$, CDDP 544.8 [403.2-1123] $\mu \mathrm{m}^{2}$; CTRL 104.6 [78-144.3] $\mu \mathrm{m}^{2}$, CDDP 98.7 [71.55-129.8] $\mathrm{m}^{2}$; CTRL 7.9 [5.25-13.5] $\mu \mathrm{m}^{2}$, CDDP 7 [4.9-10.2] $\mu \mathrm{m}^{2}$, respectively). In line with these results, previous studies performed in CDDP-treated Wistar rats demonstrated similar neuronal atrophy with a reduction in somatic, nuclear and nucleolar size (Tredici et al., 1998), confirming the strength of the animal model used in this work. In conclusion, this study shows a temporal profile of NfL levels in animals treated with platinum and taxane-based chemotherapy, and is currently the most extensive analysis of the correlation between severity of neuropathy and neuroaxonal damage, which 
might be due to the abundant presence of NfL in large-caliber myelinated axons, predominantly affected in taxane-induced peripheral neurotoxicity. These data provide further evidence to support the importance of serum NfL as reliable and easily accessible biomarkers since they correctly identified a presence of large caliber fiber degeneration in CIPN. Our findings may allow the assessment of a valid biomarker to readily detect the damage and monitor the efficacy of treatment and therefore evaluate the impact of this biomarker in neurotoxic PNS disorders.

\section{ACKNOWLEDGMENTS}

$\mathrm{GC}$ is a recipient of a research grant from Associazione Italiana Ricerca sul Cancro (AIRCProgettoIG2016Id.18631). HZ is a Wallenberg Scholar supported by grants from the Swedish Research Council (\#2018-02532), the European Research Council (\#681712), Swedish State Support for Clinical Research (\#ALFGBG-720931) and the UK Dementia Research Institute at UCL.

\section{AUTHOR CONTRIBUTIONS}

All authors made substantial contributors to design, and/or acquisition of data, and/or analysis and interpretation of data. The first draft was written by $\mathrm{CM}$, GF and GC, followed by critical revision of all authors. Individual contributors: conceived of study and design: CM, GF, GC, HZ. Performed research: all authors.......Acquisition, analysis and interpretation data: $\mathrm{KB}, \mathrm{HZ}$.....Writing the manuscript: $\mathrm{CM}, \mathrm{GF}, \mathrm{GC} \ldots$ Revising the manuscript for important intellectual content: all authors.

\section{CONFLICT OF INTEREST}

HZ has served at scientific advisory boards for Roche Diagnostics, Wave, Samumed and CogRx, has given lectures in symposia sponsored by Alzecure and Biogen, and is a co-founder of Brain Biomarker Solutions in Gothenburg AB, a GU Ventures-based platform company at the University of Gothenburg. The other authors declare that they have no conflict of interest 


\section{FIGURE CAPTIONS}

Fig1. Nerve conduction studies. The graphs show a reduction in caudal sensitive action potential amplitude from the second week of treatment until the end of treatment in PTX-treated animals. A reduction in amplitude was detected in CDDP-treated rats only at the end of the treatment. ${ }^{*} \mathrm{p}<0.05$ vs CTRL, ${ }^{* * *} \mathrm{p}<0.001$ vs CTRL (Mann-Whitney test).

Fig2. Neurofilament Light Chain (NfL) concentration. (a) Data collected indicate a progressive increase of NfL concentration in CDDP-treated group from the first week of treatment until the end of the experiment. ${ }^{*} \mathrm{p}<0.05$ vs CTRL, ${ }^{* *} \mathrm{p}<0.01$ vs CTRL, ${ }^{* * *} \mathrm{p}<0.001$ vs CTRL (Mann-Whitney test).

(b) The graph shows an important increase of NfL concentration in PTX-treated animals compared to CTRL group from the first week of treatment, which remains very high until the end of the treatment. ${ }^{* *} \mathrm{p}<0.01$ vs CTRL, $* * * p<0.001$ vs CTRL (Mann-Whitney test).

Fig3. Morphological examination of caudal nerves. Important loss of fibers and degeneration were detected in PTX-treated animals at the end of treatment indicating a severe axonopathy. Only few degenerated fibers were observed in CDDP-treated rats. Scale bar $30 \mu \mathrm{m}$. 


\section{FIGURE FILE}

Fig1

a
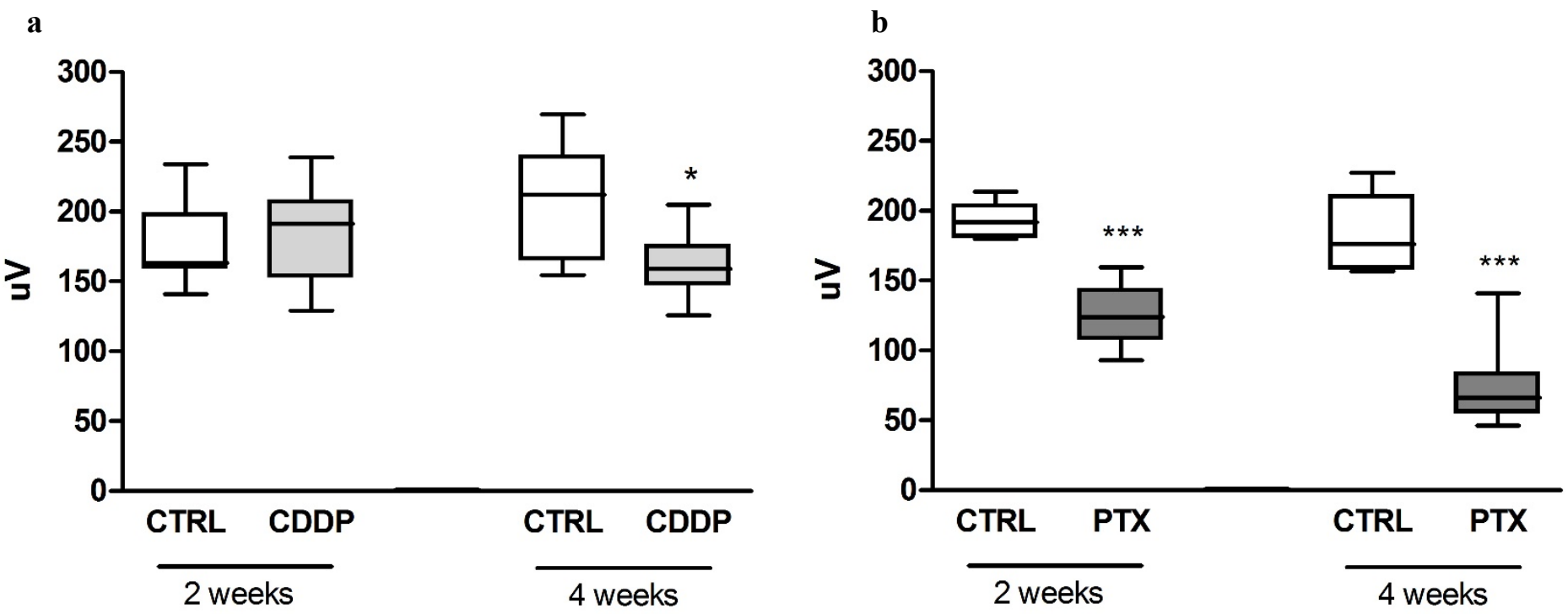

Fig2

a 


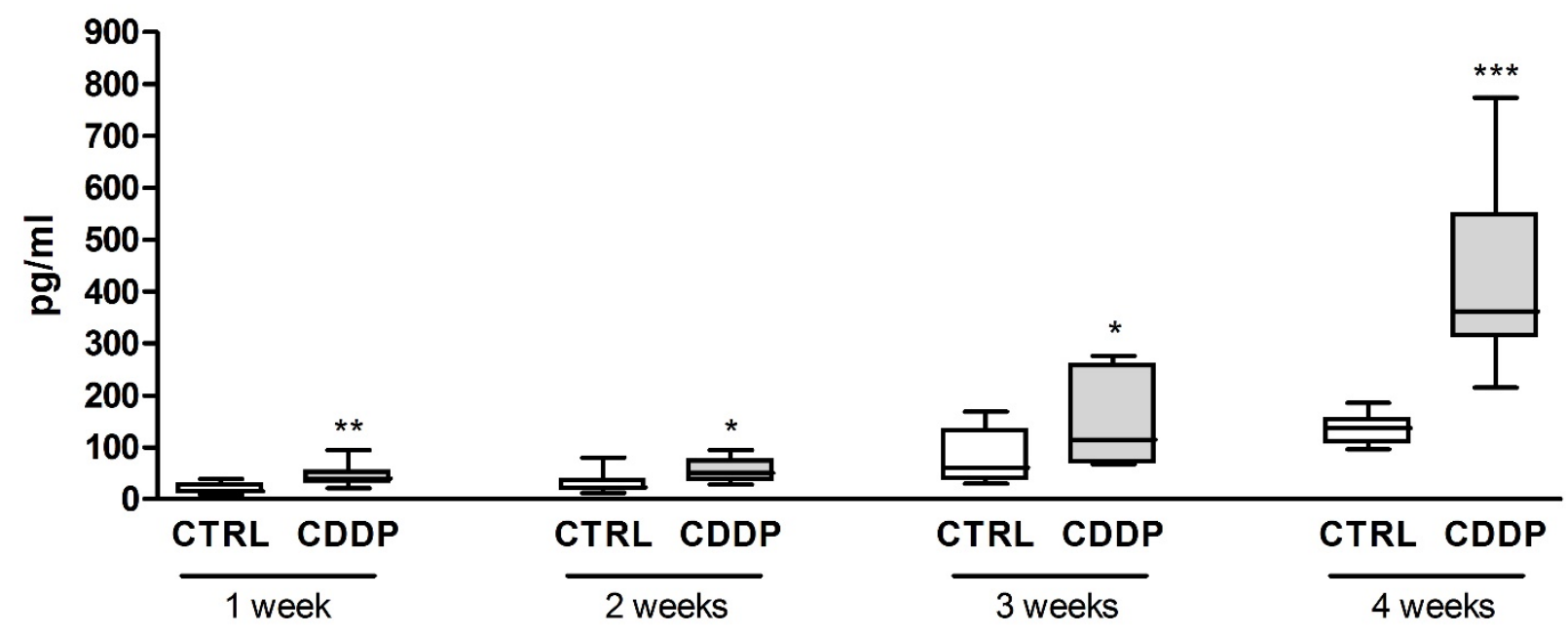

b

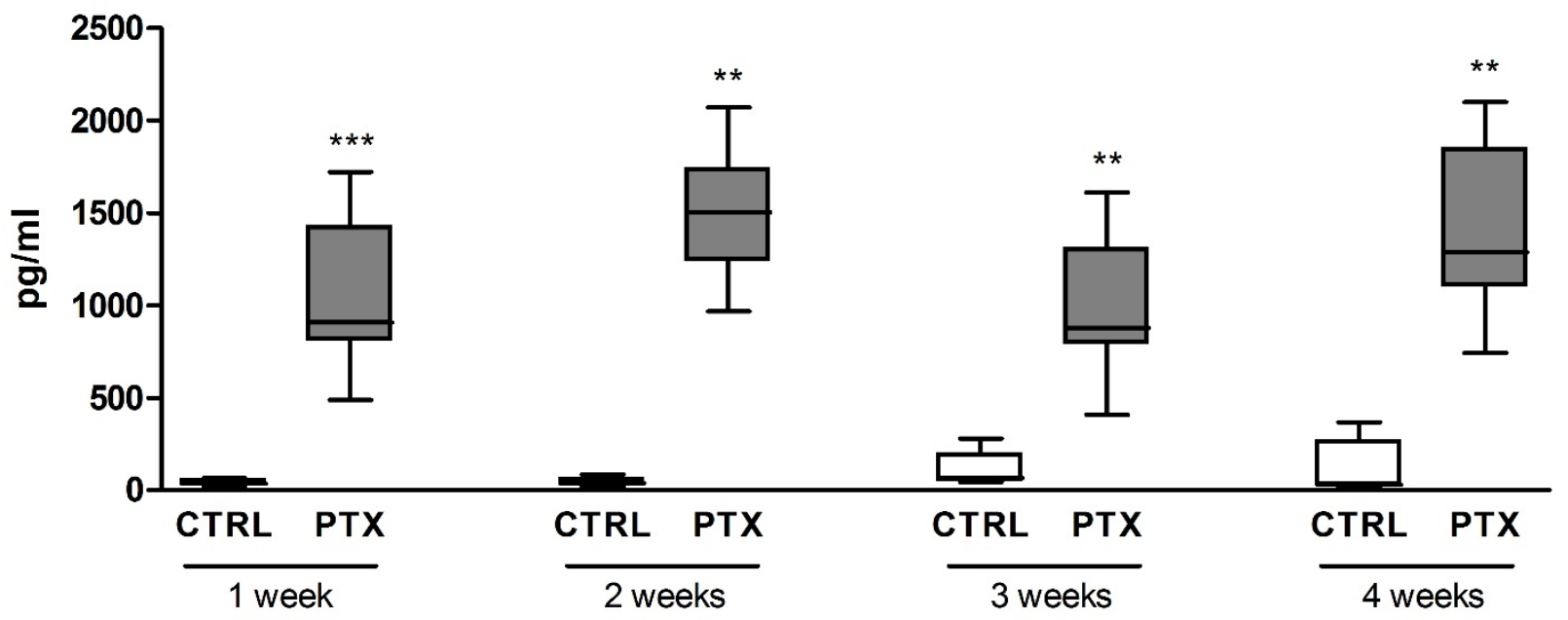

Fig3 
CTRL

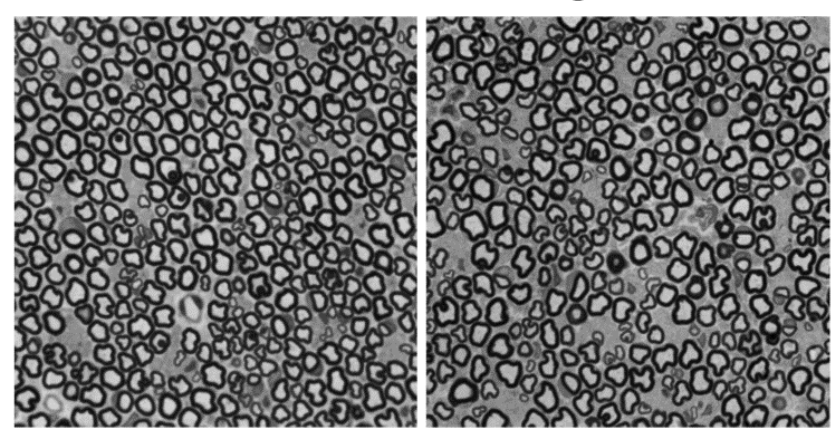

CTRL

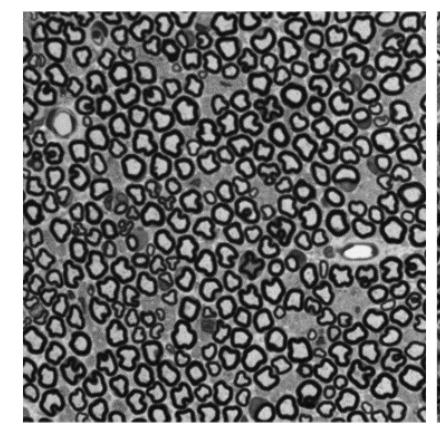

PTX



\section{REFERENCES}

Bacioglu M, Maia LF, Preische O, Schelle J, Apel A, Kaeser SA, Schweighauser M, Eninger T, Lambert M, Pilotto A, Shimshek DR, Neumann U, Kahle PJ, Staufenbiel M, Neumann M, Maetzler W, Kuhle J, Jucker M (2016) Neurofilament Light Chain in Blood and CSF as Marker of Disease Progression in Mouse Models and in Neurodegenerative Diseases. Neuron. 91(1):56-66.

Bischof A, Manigold T, Barro C, Heijnen I, Berger CT, Derfuss T, Kuhle J, Daikeler T (2018) Serum neurofilament light chain: a biomarker of neuronal injury in vasculitic neuropathy. Ann Rheum Dis. 77(7):1093-1094. doi: 10.1136/annrheumdis-2017-212045.

Brureau A, Blanchard-Bregeon V, Pech C, Hamon S, Chaillou P, Guillemot JC, Barneoud P, Bertrand P, Pradier L, Rooney T, Schussler N (2017)NF-L in cerebrospinal fluid and serum is a biomarker of neuronal damage in an inducible mouse model of neurodegeneration. Neurobiol Dis. 104:73-84. 
Byrne LM, Rodrigues FB, Blennow K, Durr A, Leavitt BR, Roos RAC, Scahill RI, Tabrizi SJ, Zetterberg H, Langbehn D, Wild EJ (2017) Neurofilament light protein in blood as a potential biomarker of neurodegeneration in Huntington's disease: a retrospective cohort analysis. Lancet Neurol. 16(8):601609.

Canta A, Chiorazzi A, Carozzi VA, Meregalli C, Oggioni N, Bossi M, Rodriguez-Menendez V, Avezza F, Crippa L, Lombardi R, de Vito G, Piazza V, Cavaletti G, Marmiroli P (2016) Age-related changes in the function and structure of the peripheral sensory pathway in mice. Neurobiol Aging.45:136-148.

Carozzi VA, Canta A, Chiorazzi A (2015) Chemotherapy-induced peripheral neuropathy: What do we know about mechanisms? Neurosci Lett. 596:90-107.

Cavaletti G, Alberti P, Marmiroli P (2015) Chemotherapy-induced peripheral neurotoxicity in cancer survivors: an underdiagnosed clinical entity? Am Soc Clin Oncol Educ Book. e553-60. b

Cavaletti G, Marmiroli P. Chemotherapy-induced peripheral neurotoxicity (2015). Curr Opin Neurol. 28(5):500-7. a

Chiorazzi A, Wozniak KM, Rais R, Wu Y, Gadiano AJ, Farah MH, Liu Y, Canta A, Alberti P, RodriguezMenendez V, Meregalli C, Fumagalli G, Monza L, Pozzi E, Vornov JJ, Polydefkis M, Pietra C, Slusher BS, Cavaletti G (2018). Ghrelin agonist HM01 attenuates chemotherapy-induced neurotoxicity in rodent models. Eur J Pharmacol. 840:89-103.

Disanto G, Barro C, Benkert P, Naegelin Y, Schädelin S, Giardiello A, Zecca C, Blennow K, Zetterberg H, Leppert D, Kappos L, Gobbi C, Kuhle J; Swiss Multiple Sclerosis Cohort Study Group (2017) Serum Neurofilament light: A biomarker of neuronal damage in multiple sclerosis. Ann Neurol. 81(6):857-870.

Khalil M, Teunissen CE, Otto M, Piehl F, Sormani MP, Gattringer T, Barro C, Kappos L, Comabella M, Fazekas F, Petzold A, Blennow K, Zetterberg H, Kuhle J (2018). Neurofilaments as biomarkers in neurological disorders. Nat Rev Neurol. 14(10):577-589.

Kuhle J, Barro C, Andreasson U, Derfuss T, Lindberg R, Sandelius Å, Liman V, Norgren N, Blennow K, Zetterberg H (2016) Comparison of three analytical platforms for quantification of the neurofilament 
light chain in blood samples: ELISA, electrochemiluminescence immunoassay and Simoa. Clin Chem Lab Med. 54(10):1655-61

Kuhle J, Gaiottino J, Leppert D, Petzold A, Bestwick JP, Malaspina A, Lu CH, Dobson R, Disanto G, Norgren N, Nissim A, Kappos L, Hurlbert J, Yong VW, Giovannoni G, Casha S (2015) Serum neurofilament light chain is a biomarker of human spinal cord injury severity and outcome. J Neurol Neurosurg Psychiatry. 86(3):273-9..

Mariotto S, Farinazzo A, Magliozzi R, Alberti D, Monaco S, Ferrari S (2018) Serum and cerebrospinal neurofilament light chain levels in patients with acquired peripheral neuropathies. J Peripher Nerv Syst. 23(3):174-177.

Mattsson N, Cullen NC, Andreasson U, Zetterberg H, Blennow K (2019) Association Between Longitudinal Plasma Neurofilament Light and Neurodegeneration in Patients With Alzheimer Disease. JAMA Neurol. 76(7):791-799.

Meregalli C, Fumagalli G, Alberti P, Canta A, Carozzi VA, Chiorazzi A, Monza L, Pozzi E, Sandelius $\AA$, Blennow K, Zetterberg H, Marmiroli P, Cavaletti G (2018). Neurofilament light chain as disease biomarker in a rodent model of chemotherapy induced peripheral neuropathy. Exp Neurol. 307:129-132.

a

Meregalli C, Marjanovic I, Scali C, Monza L, Spinoni N, Galliani C, Brivio R, Chiorazzi A, Ballarini E, Rodriguez-Menendez V, Carozzi VA, Alberti P, Fumagalli G, Pozzi E, Canta A, Quartu M, Briani C, Oggioni N, Marmiroli P, Cavaletti G (2018) High-dose intravenous immunoglobulins reduce nerve macrophage infiltration and the severity of bortezomib-induced peripheral neurotoxicity in rats. $\mathrm{J}$ Neuroinflammation. 15(1):232. b

Novakova L, Zetterberg H, Sundström P, Axelsson M, Khademi M, Gunnarsson M, Malmeström C, Svenningsson A, Olsson T, Piehl F, Blennow K, Lycke J (2017). Monitoring disease activity in multiple sclerosis using serum neurofilament light protein. Neurology. 89(22):2230-2237. 
Perrot R, Berges R, Bocquet A, Eyer J (2008). Review of the multiple aspects of neurofilament functions, and their possible contribution to neurodegeneration. Mol Neurobiol. 38(1):27-65.

Perrot R, Eyer J. Neuronal intermediate filaments and neurodegenerative disorders (2009). Brain Res Bull. 80(4-5):282-95.

Rohrer JD, Woollacott IO, Dick KM, Brotherhood E, Gordon E, Fellows A, Toombs J, Druyeh R, Cardoso MJ, Ourselin S, Nicholas JM, Norgren N, Mead S, Andreasson U, Blennow K, Schott JM, Fox NC, Warren JD, Zetterberg H (2016). Serum neurofilament light chain protein is a measure of disease intensity in frontotemporal dementia. Neurology. 87(13):1329-36.

Roytta M and Raine CS (1986) Taxol-induced neuropathy: short-term effects of local injection. J Neurocytol. 15(4):483-96.

Sandelius Å, Zetterberg H, Blennow K, Adiutori R, Malaspina A, Laura M, Reilly MM, Rossor AM (2018) Plasma neurofilament light chain concentration in the inherited peripheral neuropathies. Neurology. 90(6):e518-e524

Soylu-Kucharz R, Sandelius Å, Sjögren M, Blennow K, Wild EJ, Zetterberg H, Björkqvist M (2017) Neurofilament light protein in CSF and blood is associated with neurodegeneration and disease severity in Huntington's disease R6/2 mice. Sci Rep. 7(1):14114.

Tredici G, Tredici S, Fabbrica D, Minoia C, Cavaletti G (1998) Experimental cisplatin neuronopathy in rats and the effect of retinoic acid administration. J Neurooncol. 36(1):31-40.

Velasco R, Bruna J (2010) [Chemotherapy-induced peripheral neuropathy: an unresolved issue]. Neurologia. 25(2):116-31.

Yuan A, Rao MV, Veeranna, Nixon RA (2012) Neurofilaments at a glance. J Cell Sci. 125(Pt 14):325763. 